\title{
Destruction of Perfluoroalkyl Acids Accumulated in Typha latifolia through Hydrothermal Liquefaction
}

Weilan Zhang ${ }^{1}$, Huimin Cao ${ }^{1,2}$, Seshasayee Mahadevan Subramanya ${ }^{3}$, Phillip Savage ${ }^{3}$, Yanna Liang ${ }^{1 *}$

${ }^{1}$ Department of Environmental and Sustainable Engineering, University at Albany, SUNY, 1400

Washington Avenue, Albany, NY 12222, USA.

${ }^{2}$ College of Environmental Science and Engineering, Nankai University, No.38 Tongyan Road, Jinnan District, Tianjin, 300350, P.R. China.

${ }^{3}$ Department of Chemical Engineering, 121 Chemical and Biomedical Engineering Building, The Pennsylvania State University, University Park, PA 16802-4400

\section{* Corresponding author}

Email: yliang3@albany.edu

Tel: +1 (518) 437-4979

Address: 1400 Washington Avenue, Albany, NY 12222, USA.

Number of pages: 10

Number of figures: 2

Number of tables: 1 


\section{Text S1. Manufacturer information of reactors}

Three kinds of stainless-steel reactors with different sizes (i.e., 4-mL, 15-mL, 300-mL) were used in this study. The detailed information of the reactors is provided in the Supporting Information (SI). The 4-mL reactors were made from $1 / 2$ in. Swagelok tube fittings (a port connector and two caps) (Solon, OH, USA). The 15-mL reactors (MS-15 Micro Reactor) were purchased from High Pressure Equipment Co. (Erie, PA, USA). The 300-mL reactor (5521 HP Compact Reactor with 4848 Controller) was purchased from Parr Instrument Company (Moline, IL, USA).

\section{Text S2. Plant cultivation and sample processing}

The collected cattails were mature, around 4-ft tall, but without heads. After rinsing off soil from the plant roots completely, one plant was subject to PFAA extraction as detailed in Text S4 to obtain background PFAA concentrations in these plants. Three plants were grown hydroponically in 2-L HDPE bottles containing half strength Hoagland solution with a mixture of five PFAAs (i.e., PFOA, PFOS, PFBA, PFHpA, and PFHxA). The concentration of each PFAA in the bottle was $2,000 \mu \mathrm{g} / \mathrm{L}$. The plants were cultivated in a greenhouse and exposed to PFAAs for 2 weeks. Another three were grown as controls in the same half strength Hoagland solution without any PFAAs under the same growth condition. At the time of harvest, each plant was divided into shoots and roots which were rinsed carefully with tap water and deionized water sequentially. Clean shoots and roots were then freeze-dried at a temperature of $-40{ }^{\circ} \mathrm{C}$ for $48 \mathrm{~h}$. The freeze-dried plant tissues were then ground into powder using a coffee grinder and stored at $-80^{\circ} \mathrm{C}$. 


\section{Text S3. Hydrothermal liquefaction procedure}

Hydrothermal liquefaction (HTL) of pure PFAA solutions was performed in different reactors and run in triplicate. Concentrations of the eight PFAAs were selected according to a report published by Anderson et al. ${ }^{1}$ about the maximum concentrations of PFAS detected in groundwater collected from non-FTA sites on active U.S. Air Force installations throughout the continental United States. At these sites, the maximum concentrations are: PFBA- $64 \mu \mathrm{g} / \mathrm{L}$,

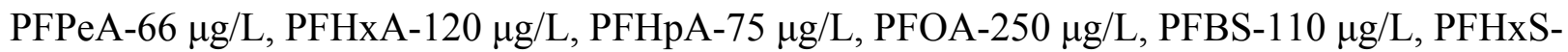
$290 \mu \mathrm{g} / \mathrm{L}, \mathrm{PFOS}-4,300 \mu \mathrm{g} / \mathrm{L}$. The concentrations of individual PFOA and PFOS in tested solutions were also set up based on this report.

For the 4-mL reactors, $2.3 \mathrm{~mL}$ of different PFAA solutions (i.e., PFOA at $200 \mu \mathrm{g} / \mathrm{L}$, PFOS at 4,300 $\mu \mathrm{g} / \mathrm{L}$, or mixtures of PFAAs) were first added. Then, the 4-mL reactors were sealed and heated at $300{ }^{\circ} \mathrm{C}$ for 2 hours. After HTL, the entire content in the reactor was flushed out by using $10 \mathrm{~mL}$ of deionized water. The subsamples were analyzed for concentrations of PFAS and fluoride.

A similar study was also conducted in a $300-\mathrm{mL}$ reactor with or without a base catalyst (i.e., $\mathrm{KOH}$ ). Briefly, one hundred $\mathrm{mL}$ of the PFAA mixture mentioned above with or without 1 $\mathrm{M}$ of $\mathrm{KOH}$ was added to the $300-\mathrm{mL}$ reactor. The reactor was then sealed, heated and kept at $300{ }^{\circ} \mathrm{C}$ for 2 hours. After HTL and the reactors reaching to room temperature, the solution in the reactor was poured out followed by thorough rinsing of the reaction chamber. The solution and the rinses were combined for analysis of PFAS.

HTL of cattail plant shoots and roots was conducted in 15-mL reactors. Around 0.09 gram of ground powder of freeze-dried cattails with or without PFAA exposures was loaded to each reactor. Afterwards, $10 \mathrm{~mL}$ of deionized water was injected into the reactor. The reactor 
was then heated at $300{ }^{\circ} \mathrm{C}$ for 2 hours. After HTL, the whole content was flushed out using 40 $\mathrm{mL}$ of deionized water and separated into a liquid phase and a solid phase by vacuum filtration with a $0.2-\mu \mathrm{m}$ filter. Both phases were subject to PFAS analyses as described in the following. It needs to be noted that phase separation of the liquid phase was not observed and was not intended for this work. Following liquid-solid separation, the entire liquid phase was subject to solid phase extraction of PFAS.

\section{Text S4. Extraction of PFAAs}

PFAAs in the aqueous solutions were analyzed following ASTM standard method D7979-17 and EPA 537.1. The water sample was mixed with methanol using a 1:1 mix ratio. Then, the mixture was vortexed and passed through a $0.2-\mu \mathrm{m}$ nylon filter. To avoid potential PFAA sorption to the filters, the first $3 \mathrm{~mL}$ of filtrate were discarded. PFAAs in the filtrate were then analyzed.

PFAAs in the freeze-dried cattails were extracted by MTBE-NaOH according to a procedure we published recently ${ }^{2}$. Briefly, five $\mathrm{mL}$ of $\mathrm{NaOH}(0.4 \mathrm{M})$ was added to a $50-\mathrm{mL}$ polypropylene tube containing the freeze-dried materials. Then, the mixture was spiked with a surrogate $\left({ }^{13} \mathrm{C}-\mathrm{PFHxA}\right)$ and homogenized using a handheld homogenizer and left in a refrigerator at $4{ }^{\circ} \mathrm{C}$ overnight. Afterwards, two $\mathrm{mL}$ of tetrabutylammonium hydrogensulfate (TBAHS, 0.5 $\mathrm{M})$ and four $\mathrm{mL}$ of $\mathrm{Na}_{2} \mathrm{CO}_{3}$ buffer $(0.25 \mathrm{M})$ were added for PFAAs extraction. After vortexing, five $\mathrm{mL}$ of tert-Butyl methyl ether (MTBE) was added to the mixture, and the mixture was shaken vigorously for $20 \mathrm{~min}$. The organic and aqueous layers were separated by centrifugation, and the MTBE layer was transferred to a second polypropylene tube. The aqueous phase was further extracted twice with $2 \times 5 \mathrm{~mL}$ MTBE. All organic fractions of the three extractions were 
combined and allowed to evaporate under a gentle stream of nitrogen before being reconstituted in $1.0 \mathrm{~mL}$ of methanol. The sample was diluted with nine $\mathrm{mL}$ water and cleaned up by a HyperSep C18 cartridge (Thermo Scientific, Waltham, MA, USA) conditioned with $10 \mathrm{~mL}$ of methanol and $10 \mathrm{~mL}$ of MTBE. PFAAs in the cartridge were then eluted by $4 \mathrm{~mL}$ methanol and $4 \mathrm{~mL}$ ammonium hydroxide ( $0.1 \%$ in methanol). The $8 \mathrm{~mL}$ eluate was evaporated under nitrogen before being reconstituted in $2.0 \mathrm{~mL}$ of methanol and internal standards: ${ }^{13} \mathrm{C}$ PFOA and ${ }^{13} \mathrm{CPFOS}$. Afterwards, the concentrations of PFAAs in the methanol solution were measured by LC/MS/MS as shown below. The extraction efficiency was obtained by calculating the surrogate recovery $(\% \mathrm{R}) . \% \mathrm{R}=(\mathrm{A} / \mathrm{B}) \times 100 \%$, where $\mathrm{A}$ is the calculated SUR concentration for the QC and B is the fortified concentration of the SUR. The exact value of the surrogate recovery in our study was $94.3 \pm 8.82 \%$.

PFAAs in the liquid phase derived from HTL of cattail tissues were extracted via a solid phase extraction process. The liquid sample was passed through a HyperSep C18 cartridge conditioned with $10 \mathrm{~mL}$ of methanol and $10 \mathrm{~mL}$ of MTBE. PFAAs in the cartridge were then eluted by $4 \mathrm{~mL}$ methanol and $4 \mathrm{~mL}$ ammonium hydroxide ( $0.1 \%$ in methanol). The $8 \mathrm{~mL}$ eluate was evaporated under nitrogen before being reconstituted in $2.0 \mathrm{~mL}$ of methanol and internal standards: ${ }^{13} \mathrm{C}$ PFOA and ${ }^{13} \mathrm{CPFOS}$. Afterwards, the concentrations of PFAAs in the methanol solution were measured by LC/MS/MS.

PFAAs in the solid phase derived from HTL of cattail tissues were extracted following a method published by Higgins et al. ${ }^{3}$. According to the report, the extraction efficiency of this method for sediments and sludge ranged from 73 to $98 \%$. In short, the solids were transferred to a $50-\mathrm{mL}$ centrifugation tube, to which $10 \mathrm{~mL}$ of acetic acid (1\%) was added. The tube was vortexed and sonicated for $15 \mathrm{~min}$ at $60{ }^{\circ} \mathrm{C}$. Afterwards, the tube was centrifuged at 4,500 rpm 
for $5 \mathrm{~min}$, and the supernatant was decanted into a second $50-\mathrm{mL}$ tube. An aliquot of the methanol (90\%)/acetic acid (1\%) extraction solvent mixture $(2.5 \mathrm{~mL})$ was then added to the original tube and the tube was again vortexed and sonicated for $15 \mathrm{~min}$ at $60^{\circ} \mathrm{C}$ before centrifuging and decanting the extract. This process of acetic acid washing followed by methanol/acetic acid extraction was repeated, and a final $10-\mathrm{mL}$ acetic acid wash was performed. For each sample, all washes and extracts were combined. The combination was cleaned up using the same HyperSep C18 cartridge mentioned above. The PFAAs in the eluents were then quantified.

\section{Text S5. Quantification of fluoride ion}

The concentration of fluoride ion in aqueous samples was measured in Water Quality Laboratory at the Pennsylvania State University following the standard method 4110 B: Ion Chromatography with Chemical Suppression of Eluent Conductivity. The model of the ion chromatography was Dionex ICS3000 (Thermo Scientific, Waltham, MA, USA). The quality control samples gave the expected results, showing the high precision and reliability of the measurement system. The recoveries of the spiked fluoride ions at $0.02 \mathrm{mg} / \mathrm{L}$ and $0.2 \mathrm{mg} / \mathrm{L}$ in quality control samples were $95 \%$ and $98.5 \%$, respectively.

\section{Text S6. Quantification of PFAAs}

Quantification of PFAAs was performed using a 1290 Infinity II LC system coupled with a 6470 Triple Quad Mass Spectrometer (Agilent Technologies, Santa Clara, CA, USA). Before analysis, internal standards, ${ }^{13} \mathrm{C}-\mathrm{PFOA}$ and ${ }^{13} \mathrm{C}$-PFOS, were added to all standards and samples to yield a concentration of $10 \mathrm{pg} / \mu \mathrm{L}$. An Agilent ZORBAX Eclipse Plus C18 $(3.0 \times 50 \mathrm{~mm}, 1.8$ 
$\mu \mathrm{m})$ analytical column and an Agilent Eclipse Plus C18 $(4.6 \times 50 \mathrm{~mm}, 3.5 \mu \mathrm{m})$ delay column were used at $50^{\circ} \mathrm{C}$. The injection volume of samples was $5 \mu \mathrm{L}$. Solvent A was $5 \mathrm{mM}$ ammonium acetate in water and solvent B was $5 \mathrm{mM}$ ammonium acetate in $95 \%$ methanol. The flow rate of the mobile phase was $0.5 \mathrm{~mL} / \mathrm{min}$. The $\mathrm{LC}$ gradient elution started at $70 \% \mathrm{~A}$, decreased to $0 \% \mathrm{~A}$ at $8 \mathrm{~min}$ and held for $4 \mathrm{~min}$ before reverting to original conditions. The MS instrument was operated in Agilent Jet Stream electrospray negative ionization mode and dynamic multiple reaction monitoring (dMRM) mode. Since the solution matrices for individual PFAS compounds and PFAA mixtures were different, for all our PFAS analysis, both surrogate and internal standards were used. To calculate concentrations of PFAS, the calibration curve we used were concentrations of the standard vs. ratio of the peak area of the analyte to the area of its corresponding internal standard.

\section{Text S7. Quality control and data analysis}

Throughout all experiments reported in this study, quality control required by EPA Method 537.1 was strictly followed. The extraction efficiency was determined by calculating the ratios of surrogate mass determined in samples to the initial spiked surrogate mass. The extraction efficiencies in all samples were all above $90 \%$. The statistical analysis of the experimental data was performed by mean of one-way ANOVA using IBM SPSS Statistics 22. Duncan test was conducted for post-hoc comparisons. Student $t$ test was conducted to determine the significance of differences between two sets of data. Statistical significance was accepted when $\mathrm{p}<0.05$. 
Table S1. Chemical reagents used in this study.

\begin{tabular}{lll}
\hline Substance & Grade/Purity & Supplier \\
\hline \hline Perfluoropentanoic acid & $\geq 97 \%$ & Fisher scientific \\
Perfluorohexanoic acid & $\geq 98 \%$ & Fisher scientific \\
Perfluoroheptanoic acid & $\geq 98 \%$ & Fisher scientific \\
Perfluorooctanoic acid & $\geq 96 \%$ & Sigma-Aldrich \\
Potassium perfluorobutanesulfonate & $\geq 98 \%$ & Fisher scientific \\
Perfluorohexanesulfonic acid potassium salt & $\geq 98 \%$ & Fisher scientific \\
Heptadecafluorooctanesulfonic acid potassium salt & $\geq 98.0 \%$ & Sigma-Aldrich \\
Perfluoropentanoic acid & $\geq 97 \%$ & Fisher scientific \\
& $\geq 98 \%$ & Wellington \\
Perfluoro-n-[1,2- $\left.{ }^{13} \mathrm{C}_{2}\right]$ octanoic acid & & Laboratories Inc. \\
& $\geq 98 \%$ & Wellington \\
Sodium perfluoro-1[1,2,3,4- $\left.{ }^{13} \mathrm{C}_{4}\right]$ octanesulfonate & Laboratories Inc. \\
Sodium hydroxide & Certified ACS & Fisher scientific \\
Sodium Carbonate Anhydrous & Certified ACS & ACROS Organics \\
Tetrabutylammonium hydrogensulfate & Certified ACS & ACROS Organics \\
tert-Butyl methyl ether & LC/MS Grade & Fisher scientific \\
Ammonium acetate & LC/MS Grade & Fisher scientific \\
Methanol & LC/MS Grade & Fisher scientific \\
Water & N/A & Thermo Scientific \\
HyperSep C18 cartridge & N/A & Thermo Scientific \\
2.0-mL PP vials and caps for LC
\end{tabular}




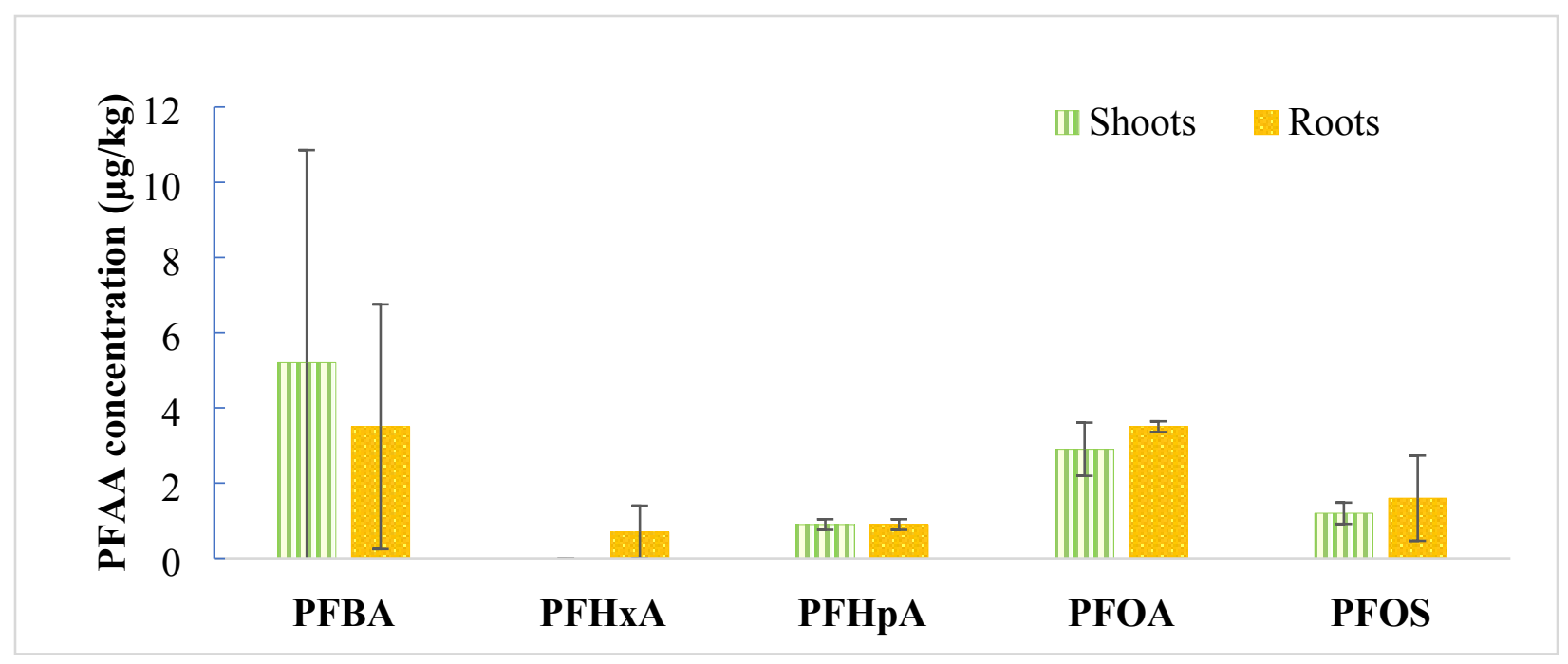

Fig. S1: PFAA concentrations in cattails without exposure to these compounds.

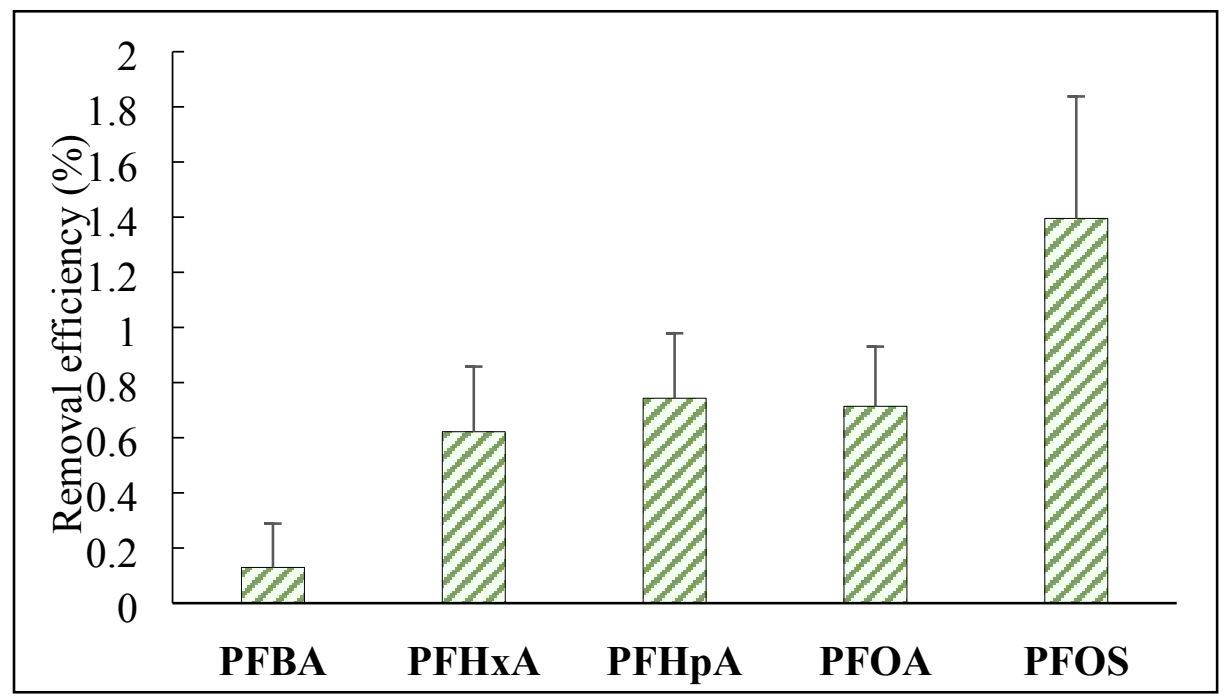

Fig. S2: PFAA removal efficiencies by cattails in the hydroponic system. The removal efficiency was calculated by dividing the PFAA mass in plant tissues by the initial PFAA mass spiked in the growth medium. 


\section{References}

1. Anderson, R. H.; Long, G. C.; Porter, R. C.; Anderson, J. K., Occurrence of select perfluoroalkyl substances at US Air Force aqueous film-forming foam release sites other than fire-training areas: Field-validation of critical fate and transport properties. Chemosphere 2016, 150, 678-685, DOI: 10.1016/j.chemosphere.2016.01.014.

2. Zhang, W.; Zhang, D.; Zagorevski, D. V.; Liang, Y., Exposure of Juncus effusus to seven perfluoroalkyl acids: Uptake, accumulation and phytotoxicity. Chemosphere 2019, 233, 300-308, DOI: 10.1016/j.chemosphere.2019.05.258.

3. Higgins, C. P.; Field, J. A.; Criddle, C. S.; Luthy, R. G., Quantitative determination of perfluorochemicals in sediments and domestic sludge. Environ. Sci. Technol. 2005, 39 (11), 3946-3956, DOI: 10.1021/es048245p. 